\title{
A supply chain management model to optimise the sorting capability of a 'third party logistics' distribution centre
}

\author{
V.S.S. Yadavalli* and C. Balcou \\ *To whom all correspondence should be addressed \\ Sarma.Yadavalli@up.ac.za
}

Department of Industrial and Systems Engineering, University of Pretoria, South Africa

\begin{abstract}
In this paper, the process flow within a high-end 3PL distribution centre is studied in order to determine the optimal split between the parcel size categories moved within the system. The article considers the application of an optimisation (MIQP) model with a corresponding discrete event simulation model to validate the authenticity of the solution. The study assumes that the current process flow and layout of the centre remains fixed; the entering and exiting routes to and from the centre and the centre itself are located optimally within the network and the models have base times of one and seven days respectively. Performance measures have been analysed through utilization states of equipment and labour, queue contents versus time and average throughput times of each of the parcel categories. The findings in this paper can create a positive trigger for improvement within the distribution centre as equipment and labour can be downsized to decrease costs and improve safety and benefit the company through the alignment of a network of distribution centres that each applies modified relevant versions of the modelling logic conveyed in this paper.
\end{abstract}

\section{Introduction}

A 3PL is defined as an: "arrangement in which a firm with long and varied supply chains outsources its logistical operations to one or more specialist firms, the third party logistics providers," (Business Dictionary, 2015). Companies have taken a new view on the process of outsourcing as one that allows the company to concentrate on their core business resulting in improved performance and reduced costs.

A study performed in the US by (Lieb \& Bentz, 2005: 5-12) found that roughly $60 \%$ of the Fortune 500 companies reported that the company held at least one third party logistics (3PL) contract, however a more recent report released by (Armstrong \& Associates, 2013) concluded that there has been a $67 \%$ increase in the 3PL market of the Fortune 500 companies since 2005 showing the staggering increase of growth in the transportation and logistics industry. This growth leads to the increased need for a company to differentiate themselves from competitors operating in the same market and find improved ways to increase customer satisfaction while simultaneously reducing costs. The requirement for a company to look in-house for ways to achieve this goal is therefore becoming more apparent and the problem analysed in this study arises. Due to the nature of the 3PL industry and the versatility of the client base using the services of a distribution centre, it is difficult to accurately predict the trend in demand. There is therefore a lack of transparency with respect to how frequently and how much of each parcel category size will need to be processed by the centre on a daily basis. This uncertainty leads to periodic bottlenecks that occur as a result of the operational process.
In the long run this problem can become detrimental if the lack of transparency becomes a gap in the ability to continuously deliver on customer expectations and prescribed service level agreements. Determining an optimal parcel category split therefore has the benefits of ensuring that parcels are processed efficiently and effectively to bridge the potential gap.

The problem of determining the optimal split becomes unique as a result of the process flow relevant to the studied distribution centre. Related works are therefore scarce. The Parcel Hub Scheduling Problem (PHSP) in which the primary objective is finding an unload schedule that will minimize the duration of the transferring operation (unloading of vehicles and transferring these sorted loads to their required loading docks) within a terminal (distribution centre), has gained considerable interest since its inception. The PHSP first comes up in literature in the simulation-based study (Masel \& Goldsmith, 1997) in which the time span and congestion of the transfer operation within a distribution centre is evaluated by assigning load docks to specific destinations. Research into the PHSP gained its largest advance through the studies proposed by (McWilliams, Stanfield \& Geiger, 2005: 393412) and in the later works by the same authors (McWilliams, et al., 2008: 709-720) which provides research in the form of a simulation optimization algorithm to solve the PHSP. The studies propose a set of assumptions, but could not be used to directly solve the problem in this study as the assumptions did not coherently align. In particular this study uses multiple transporting methods within the distribution centre whereas the PHSP only considers conveyor belts. Secondly, trucks do not necessarily have equal batch sizes, as assumed in the PHSP, as this is dependent on the demand from the inbound 
locations in this study. Furthermore, the PHSP is focused on minimization of transfer times and although this is relevant this study uses a base time of 24 hours and is aiming to maximize the throughput during that time period, hence the notion of time will not necessarily aid in this study's objective.

In order to establish an optimal parcel category capacity split to distinguish the number of each of the four parcel categories analysed in this paper, two models were required: the first to find the split and the second to validate the first's results. An optimisation model which falls under the field of Operations Research was elected as the primary choice of modelling logic due to the advantages attributed to this type of model. A few of the more predominant reasons for the decision are in line with the benefits of Operations Research Modelling as quoted in the paper "Operations Research" by (Tiwari \& Shandilya, 2006) which includes the scientific approach with which Operations Research models are predefined allowing the modeller to achieve the accurate generation of results due to the deterministic methods that are used to obtain a solution. Furthermore, these models allow the modeller to portray a better co-ordination and planning of the constraints and other factors that influence the operations within a modelled scenario, in this case a distribution centre.

Although the application of Operations Research can become complex and at times lengthy, it is important to continue to make various iterations of the model so as to refine the logic. This iterative process was completed in the course of this study where the original optimisation model was split into two smaller models to reduce both run time and complexity within the model.

A simulation model was then elected as a second validating method for the completed optimisation model. The benefits of simulation are ten-fold, but the predominant contributors that favour this modelling logic are outlined by (Robinson, 2014) which includes the ability to model variability, interconnected components or sub-systems and complexity. These are important aspects when trying to validate the results from a corresponding type of model. In this study the distribution centre has a wide range of variability ranging from the changing arrival schedules on a daily basis to the processing time of each of the parcel types within the system. In addition there are multiple components that are interconnected within the model which is evident by the number of linking lines required in the working mode of the simulation; this in turn leads to increased complexity. The coding to ensure that a parcel enters the system, is processed and then leaves the system via the correctly allocated sink becomes a complex logic. In addition to Robinson's outlined benefits, simulation modelling has a vast potential to save costs and time when compared to performing the changes in real-life. Furthermore, simulation enables the modeller to apply less restrictive assumptions and provide increased transparency as a result of allowing the modeller to simulate the distribution centre as close to the real operations as is possible.
Lastly, both of these selected model options have software packages available to increase the potential of obtaining adequate results using the improved computational ability of the software.

The optimisation model was constructed in line with an interpretation of the main steps summarized by (El-Halwagi, 2006: 290-300) which includes: (1) the determination of the objective function, (2) the development of a strategy with which to develop a solution, (3) development of the constraints that limit the strategy and (4) improvement of the model formulation through iterations. The simulation model was then constructed following the process outlined by (Maria, 1997) which in a broad outline includes the identification, formulation and development of a model using collected real system data and then validating the model through appropriate experiments and interpretation of the collected results.

Currently the studied distribution centre is capable of effectively collecting, sorting and delivering the demand of each of the four parcel category sizes processed by the studied centre on a daily basis. However, due to the aforementioned difficulty to accurately predict the trend in demand, the company lacks visibility of how frequently and how much of each parcel category size will need to be processed in the system on a daily basis. If we consider the sorting process within the distribution centre, the flow of parcels is dependent on the arrival rates of these parcels.

Currently, the parcels tend to arrive in the afternoon period and thus the flow of the sorting process is highly condensed during this period but substantially decreases during the morning period. Idle time of the equipment and staff is seen to be wasted profits; however the current operations do not allow the system to shift the schedules to help to alleviate this bottleneck. Additionally, the company does not know what the optimal parcel category capacity split is that can be handled effectively and efficiently by the system and hence the problem of this study is highlighted. The company cannot take on additional clients to make up for the idle time, because it is unknown whether or not this additional capacity can be accommodated by the system. The result of the "AS-IS" state of operations on the company can be detrimental in the long run.

The 3PL industry, as highlighted repeatedly throughout literature by authors and corporations alike such as (Briggs, Landry \& Daugherty, 2010: 640-649), (Chu \& Wang, 2012: 78-96), (Eyefortransport, 2013), (Eyefortransport, 2015); is a highly competitive environment with many different criteria used when selecting a 3PL provider. The growing and evolving list of criteria highlight the fact that the slightest inability is viewed from a customer perspective as the lack of a quality service, which is ranked as the top criteria when choosing a 3PL provider. The company therefore has a growing gap that needs to be effectively analysed and through the use of the fields of Operations Research and Simulation Modelling this study will attempt to provide a means of information to bridge this growing gap in operational success. 


\section{Model description}

A number of assumptions were made in this study in order to simplify the complex environment of the problem and to enable a better understanding of the context that this study is focused on. These assumptions have been listed below.

- The retail distribution functions of the studied distribution centre were not included in this study.

- All of the amounts have been converted to volumetric kilograms (using a conversion factor of 4000) as this is the base unit used by the company to invoice their clients.

- A maximization problem rather than a minimization problem has been used to model the process as the maximum throughput is sought.

- The location of the distribution centre is fixed and assumed to be optimal with respect to its geographic location.

- The incoming and outgoing routes are fixed and necessary in order to satisfy the current and future demand.

- The routes are made up of multiple stops that have been grouped to minimize the size of the model required. Therefore, the demand of a route represents the total amount of all the stops along that route. The routes have been allocated as incoming

- The demand for each of the parcel category sizes is assumed to be the average amount of each parcel category size historically transported along each of the incoming and outgoing routes.

- The model will run over the base time period of one day ( 24 hours) in order to better portray the operations of the distribution centre as well as to minimize the effects of large volumes that would be expected to accumulate over a longer length of time.

The profit function is purely used as a means to maximize the volume of each of the parcel category sizes and therefore the optimal solution will remain the same regardless of whether or not the fixed costs are included as the same cost will always be deducted from the profit. Thus, only the variable costs have been included.

The company has developed a fixed network in which parcels are couriered. The network is made up of six hubs, twenty main centres and numerous regional towns. The company has allocated each of the regional towns in their network to a main centre and in turn each of the main centres has been allocated to a hub. An example of the regional town allocation is given in Figure 1 for the main centre in Port Shepstone.
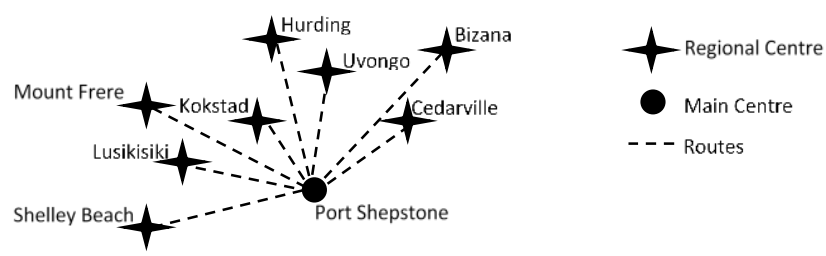

Figure 1: Regional routes converge into a main centre (This figure is only a representation and is therefore not drawn to scale).
The demands of each of the regional towns have been summed along with the demand of the main centre to represent the total demand of each of the twenty main centres across South Africa. The focus of this study is on a Johannesburg distribution centre, which forms a hub within the network as portrayed in Figure 2. The routes analysed are thus represented by the black and red dashed lines feeding into the Johannesburg hub.

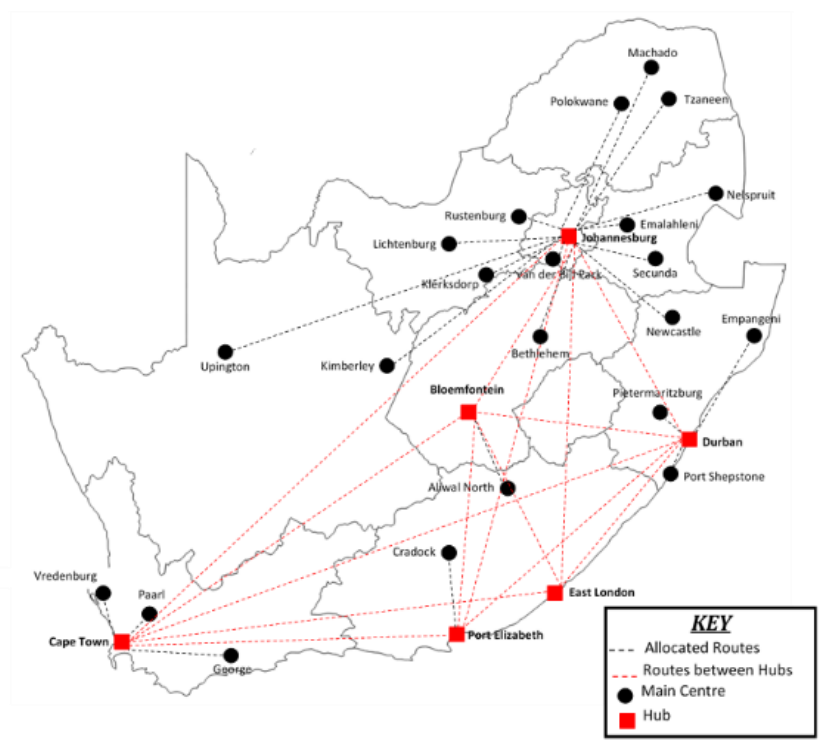

Figure 2: Company's distribution network

The black and red dashed lines that do not feed directly into the Johannesburg hub have been grouped to the closest hub from which they are serviced. As an example: Empangeni, Pietermaritzburg and Port Shepstone each have a demand that is serviced by the Durban hub. Therefore the demands for these locations to/from the Johannesburg hub are summed and represented by an incoming (Johannesburg to Durban) and outgoing (Durban to Johannesburg) demand at the Durban hub. This concept is repeated for the Paarl, Vredenburg and George centres which are summed and added to the demand of the Cape Town hub; the Cradock centre whose demand is added to that of the Port Elizabeth hub and the Aliwal North main centre whose demand is added to the Bloemfontein hub. The main centres in the network are used in order to represent the required demand from the company's client base that needs to be met.

The model needs to maximize the volume (in volumetric kilograms) that the Johannesburg distribution centre can both receive and deliver according to the transportation capacity of the fleet at the Johannesburg hub's disposal, while simultaneously meeting the required demand. The fleet is allocated according to the demand required on the route and therefore trucks are not allocated to specific routes, but rather a fleet of trucks allocated to the network flowing into and out of the Johannesburg hub.

In addition to these routes, we are required to look at the maximum capacity that can be handled within the Johannesburg hub with respect to the available equipment. 
There are four different parcel size categories, namely small, beltable, manual incompatible and forklift incompatible which are handled by the manual small sort, conveyor belts, labour and forklifts respectively. Although each of these parcel types are sometimes moved using equipment types not specifically allocated for it, we will assume for the purposes of this study that each parcel category is handled by a single equipment type as outlined previously.

Multiple iterations of defining and redefining the constraints of the model produced the original mathematical model. In this model a maximization of profits associated with transporting the four different parcel category sizes both to and from the distribution centre using one of the three service types is sought to obtain the maximum volume of incoming and outgoing parcels. The original model produced an MIQP that could be programmed and solved using the LINDO Lingo Software. The running of this model on the chosen software identified that a feasible solution is obtainable for the MIQP, however although this original model produced results for small sample sizes in a reasonable proportion of time, at full scale the model took exponentially long to solve, showing that high computational capabilities are required to solve the model. This original model was run for approximately eight hours using the Lingo software on the global solver and was interrupted to produce a local optimum in order to obtain a general idea of the trends in the solution.

Step four according to (El-Halwagi, 2006) in the formulation of an Operations Research model is to improve the formulation of the mathematical model and hence, in order to find a better solution (global optimum), the original mathematical model was broken down into two smaller submodels that maximizes the volume of each of the parcel size categories on each of the available routes and then assigns trucks to ensure that the required volume can be moved along each of the routes by maximizing the volumes loaded onto a truck as given under the next two sub-sections.

\section{Model to maximise parcel size category volumes}

Let:

I be the set of incoming routes, where $\mathrm{i}=\{1-20\}$

$\mathrm{J}$ be the set of outgoing routes, where $\mathrm{j}=\{1-20\}$

$\mathrm{P}$ be the set of parcel categories, where $\mathrm{p}=\{1-4\}$

$\mathrm{T}$ be the set of transport modes, where $\mathrm{t}=\{1-6\}$

\section{Decision variables}

$\mathrm{X}_{\mathrm{ip}} \triangleq$ the amount (in volumetric kilograms per day) of parcel size category $\mathrm{p}$ transported along route $\mathrm{i}$, where $\mathrm{p} \in \mathrm{P}$ and $i \in I$

$\mathrm{y}_{\mathrm{jp}} \triangleq$ the amount (in volumetric kilograms per day) of parcel size category $p$ transported along route $j$, where $p \in P$ and $\mathrm{j} \in \mathrm{J}$

\section{Parameters}

$\mathrm{d}_{\mathrm{ip}} \triangleq$ the given demand (in volumetric kilograms per day) for transporting parcel category size $\mathrm{p}$ along route $\mathrm{i}$, where $p \in P$ and $i \in I$

$\mathrm{n}_{\mathrm{t}} \triangleq$ the given number (in units) of transport mode $\mathrm{t}$ available, where $t \in T$

$\mathrm{d}_{\mathrm{jp}} \triangleq$ the given demand (in volumetric kilograms per day) for transporting parcel category size $\mathrm{p}$ along route $\mathrm{j}$, where $p \in P$ and $j \in J$

$\mathrm{m}_{\mathrm{p}} \triangleq$ the given maximum capacity (in volumetric kilograms per day) of parcel category size $\mathrm{p}$ that can be sorted, where $\mathrm{p} \in \mathrm{P}$

$c_{t} \triangleq$ the given maximum capacity (in volumetric kilograms) that can be transported using transport mode $\mathrm{t}$, where $\mathrm{t} \in \mathrm{T}$

\section{Objective function}

$\max \mathrm{z}=\sum_{\mathrm{p}=1}^{4}\left[\sum_{\mathrm{i}=1}^{20}\left(\mathrm{x}_{\mathrm{ip}}\right)+\sum_{\mathrm{j}=1}^{20}\left(\mathrm{y}_{\mathrm{jp}}\right)\right]$

subject to:

Incoming demand limit

$\mathrm{x}_{\text {ip }} \geq \mathrm{d}_{\text {ip }} \quad \forall \mathrm{i} \in \mathrm{I}, \mathrm{p} \in \mathrm{P}$

Outgoing demand limit

$\mathrm{y}_{\mathrm{jp}} \geq \mathrm{d}_{\mathrm{jp}} \quad \forall \mathrm{j} \in \mathrm{J}, \mathrm{p} \in \mathrm{P}$

Flow limit

$\sum_{\mathrm{i}=1}^{20} \mathrm{x}_{\mathrm{ip}}=\sum_{\mathrm{j}=1}^{20} \mathrm{y}_{\mathrm{jp}} \quad \forall \mathrm{p} \in \mathrm{P}$

Transport limit

$\sum_{\mathrm{p}=1}^{4}\left[\sum_{\mathrm{i}=1}^{20}\left(\mathrm{x}_{\mathrm{ip}}\right)+\sum_{\mathrm{j}=1}^{20}\left(\mathrm{y}_{\mathrm{j}}\right)\right] \leq \sum_{\mathrm{t}=1}^{6}\left(\mathrm{n}_{\mathrm{t}}\right)\left(\mathrm{c}_{\mathrm{t}}\right)$

Equipment capacity limit

$\sum_{\mathrm{i}=1}^{20} \mathrm{x}_{\mathrm{ip}} \leq \mathrm{m}_{\mathrm{p}} \quad \forall \mathrm{p} \in \mathrm{P}$

$\mathrm{x}_{\mathrm{ip}} \geq 0 \quad \forall \mathrm{i} \in \mathrm{I}, \mathrm{p} \in \mathrm{P} \quad$ (non-negativity)

$\mathrm{y}_{\mathrm{jp}} \geq 0 \quad \forall \mathrm{i} \in \mathrm{I}, \mathrm{p} \in \mathrm{P} \quad$ (non-negativity)

\section{Description}

The amount of each of the four parcel categories that can be handled by the Distribution Centre is maximized by the objective function in equation [1]. Equations [2] and [3] ensure that the incoming demand and outgoing demand respectively is met and Equation [4] that the volume of each of the parcel categories coming into the Distribution centre is equal to the volume of each of the parcel categories leaving the Distribution Centre. The transportation capacity limit is adhered to in equation [5] and the equipment processing limit $\mathrm{n}$ equation [6]. Non-negativity is ensured in equations [7] and [8]. 


\section{Model to assign trucks to meet demand}

Let:

I be the set of incoming routes, where $i=\{1-20\}$

$\mathrm{J}$ be the set of outgoing routes, where $\mathrm{j}=\{1-20\}$

$\mathrm{P}$ be the set of parcel categories, where $\mathrm{p}=\{1-4\}$

$\mathrm{T}$ be the set of transport modes, where $\mathrm{t}=\{1-190\}$

\section{Decision variables}

$\mathrm{x}_{\mathrm{it}} \triangleq$ the amount (in volumetric kilograms per day) of parcel size category $p$ transported along route $i$, where $p \in P$ and $i \in I$

$\mathrm{y}_{\mathrm{jt}} \triangleq$ the amount (in volumetric kilograms per day) of parcel size category $\mathrm{p}$ transported along route $j$, where $p \in P$ and $j \in J$

$\mathrm{A}_{\mathrm{it}} \triangleq\left\{\begin{array}{c}1 \text { if transport mode } \mathrm{t} \text { is used on route } \mathrm{i} \text {, } \\ \text { where } \mathrm{t} \in \mathrm{T} \text { and } \mathrm{i} \in \mathrm{I} \\ 0 \text { otherwise }\end{array}\right.$

$\mathrm{B}_{\mathrm{jt}} \triangleq\left\{\begin{array}{c}1 \text { if transport mode } \mathrm{t} \text { is used on route } \mathrm{j}, \\ \text { where } \mathrm{t} \in \mathrm{T} \text { and } \mathrm{j} \in \mathrm{J} \\ 0 \text { otherwise }\end{array}\right.$

\section{Parameters}

$\mathrm{d}_{\mathrm{i}} \triangleq$ the given demand (in volumetric kilograms per day) on route $i$, where $p \in P$ and $i \in I$

$\mathrm{d}_{\mathrm{j}} \triangleq$ the given demand (in volumetric kilograms per day) on route $j$, where $p \in P$ and $j \in J$

$c_{t} \triangleq$ the given maximum capacity (in volumetric kilograms) that can be transported using transport mode $t$, where $t \in T$

$\mathrm{n}_{\mathrm{t}} \triangleq$ the given number (in units) of transport mode $\mathrm{t}$ available, where $\mathrm{t} \in \mathrm{T}$

\section{Objective function}

$\max \mathrm{z}=\sum_{\mathrm{t}=1}^{190}\left[\sum_{\mathrm{i}=1}^{20}\left(\mathrm{~A}_{\mathrm{it}}\right)\left(\mathrm{x}_{\mathrm{it}}\right)+\sum_{\mathrm{j}=1}^{20}\left(\mathrm{~B}_{\mathrm{jt}}\right)\left(\mathrm{y}_{\mathrm{jt}}\right)\right]$

subject to:

Incoming demand limit:

$\sum_{\mathrm{t}=1}^{190}\left(\mathrm{~A}_{\mathrm{it}}\right)\left(\mathrm{x}_{\mathrm{it}}\right)=\mathrm{d}_{\mathrm{i}} \quad \forall \mathrm{i} \in \mathrm{I}$

Outgoing demand limit:

$\sum_{\mathrm{t}=1}^{190}\left(\mathrm{~B}_{\mathrm{jt}}\right)\left(\mathrm{y}_{\mathrm{jt}}\right)=\mathrm{d}_{\mathrm{j}} \quad \forall \mathrm{j} \in \mathrm{J}$

Assign a truck once

$\sum_{\mathrm{i}=1}^{20} \mathrm{x}_{\mathrm{it}}+\sum_{\mathrm{j}=1}^{20} \mathrm{y}_{\mathrm{jt}} \leq 1 \quad \forall \mathrm{t} \in \mathrm{T}$

Incoming transport capacity

$\sum_{\mathrm{i}=1}^{190} \mathrm{x}_{\mathrm{it}} \leq \mathrm{c}_{\mathrm{t}} \quad \forall \mathrm{t} \in \mathrm{T}$

Outgoing transport capacity

$\sum_{\mathrm{i}=1}^{190} \mathrm{y}_{\mathrm{jt}} \leq \mathrm{c}_{\mathrm{t}} \quad \forall \mathrm{t} \in \mathrm{T}$

$\mathrm{x}_{\mathrm{ip}} \geq 0 \quad \forall \mathrm{i} \in \mathrm{I}, \mathrm{t} \in \mathrm{T} \quad$ (non-negativity)

$\mathrm{y}_{\mathrm{jp}} \geq 0 \quad \forall \mathrm{j} \in \mathrm{J}, \mathrm{t} \in \mathrm{T} \quad$ (non-negativity)

$\mathrm{A}_{\mathrm{it}} \in\{0,1\} \quad \forall \mathrm{i} \in \mathrm{I}, \mathrm{t} \in \mathrm{T} \quad$ (binary variable)

$\mathrm{B}_{\mathrm{jt}} \in\{0,1\} \quad \forall \mathrm{j} \in \mathrm{J}, \mathrm{t} \in \mathrm{T} \quad$ (binary variable)

\section{Description}

The amount that is sent on each of the trucks at the disposal of the Distribution Centre is maximized by the objective function in equation [1]. Equations [2] and [3] ensure that the incoming demand and outgoing demand respectively is met. Equation [4] ensures that each truck is only assigned once on both incoming and outgoing routes. The transportation capacity limit is adhered to in equations [5] and [6] for incoming and outgoing routes respectively. Non-negativity is ensured in equations [7] and [8] and the use of binary variables in equations [9] and [10].

\section{Findings}

The optimisation models established the optimal percentage split depicted in Figure 3 which was compared to the current split depicted in Figure 4 as analysed for the distribution centre in this study. Although the results found for the studied centre shows a drastic increase in beltable parcels, as is expected due to the high level of automation involved in the processing of this category, the changes are dependent on the capabilities of the distribution centre analysed.

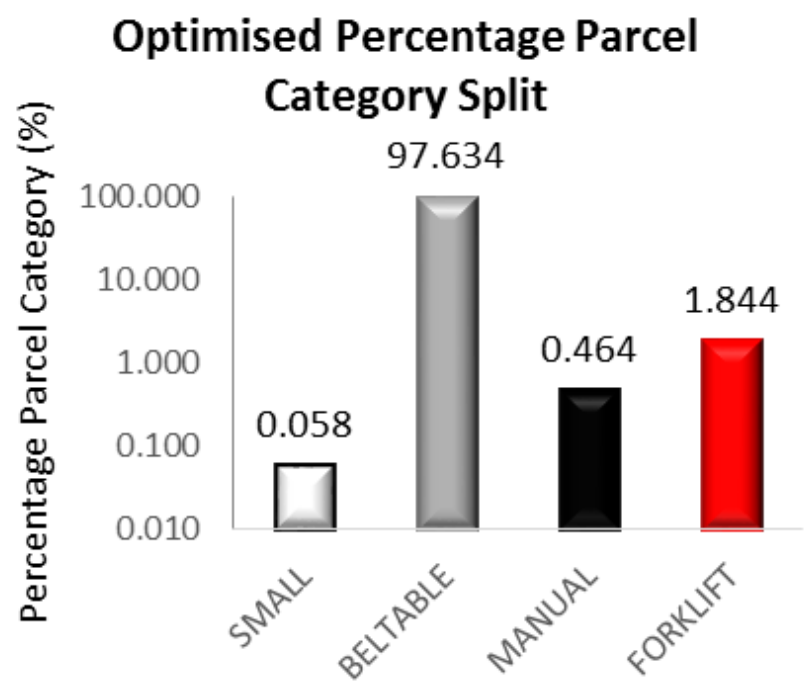

Figure 3: Analysis of the optimal parcel category percentage split

The objective of this study was established through the optimisation models and produced results which enable increased visibility of the process and related constraints within a distribution centre. 


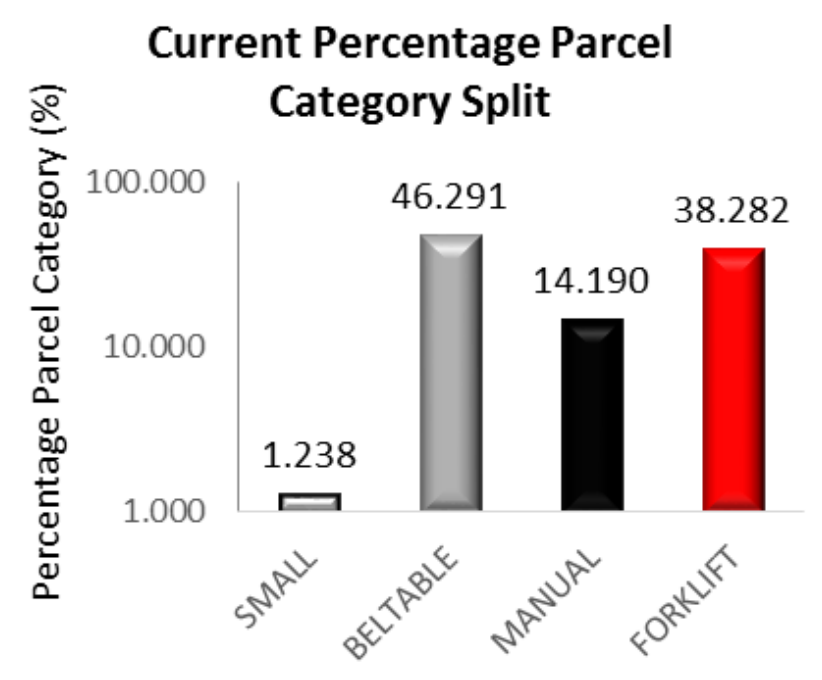

Figure 4: Analysis of the current parcel category percentage split

The established optimal split was then validated using the constructed discrete-event simulation model using the Flexsim Software. The most important performance criteria that was analysed each of the scenarios constructed in the simulation model has been listed below:

- Equipment Utilization States.

- Forklift Utilization States.

- Worker Utilization States.

- Content versus Time of the Outbound Queues.

- Content versus Time of the Primary Sort.

- Throughput Time of Each Parcel Type.

These criteria were recorded using dashboards on the simulation software which were used to compare the results obtained under the current and optimal parcel category percentage splits and to aid in the establishment of selective changes which could lead to the improvement of the current processes.

The first and most apparent of these is the increased volumes of the beltable parcel size category and decreased volumes of the other three, allowing for increased productivity levels and overall throughput times as the beltable category follows a highly automated process within the distribution centre. However, this study provides a very important distinction that although there are significant decreases in the other three parcel size categories; none of these are completely removed from the business provisions of the company. Having the ability to move all four of these parcel size categories provides the company with a differentiation factor in the marketplace, which is an important aspect of competitive advantage.

The second change became evident when analysing alternatives with respect to the number of forklifts and required labour workforce in runs of the simulation model in which the removal of three of the five forklifts and $20 \%$ of the current workforce provided low significant impacts on the utilization, queuing contents and throughput times in the distribution centre. This means that significant cost reductions could be made through the selling of three forklifts, the decreased maintenance costs of the remaining two forklifts, and the decreased labour costs from the reduction of the workforce needed. In addition safety factors are likely to increase as there is less risk of injury with fewer forklifts and workers in the busy distribution centre.

The third positive outcome from this study is the ability to implement similar solutions to any of the company's distribution centres. The key differences would be in the data entries and origin and destination centres entering and exiting from the analysed centre. These minor changes can be made and entered into the constructed optimisation model and a new optimal percentage split according to the analysed distribution centre could be obtained.

The simulation model would require a more complex understanding and multitude of changes to achieve an adequate validation process as was used in this study. This is due to the multiple changes from one centre to another including, but not limited to: the changing layouts, processes and input data. However, the modelling logic can certainly be used to cut construction times of the models relevant to these centres. In addition the performance criteria dashboards could still be applied by changing the processes/queues that are analysed.

\section{Validating the models}

Validation is the process of checking the accuracy of the model with regards to the real system and the simulation thereof (Sargent, 2011). In order to validate the formulated optimisation model, a comparative analysis can be made between the current percentage split and the formulated optimal percentage split. Although this will provide insight into the logical differences/similarities in the parcel category percentage split it is not one that invokes confidence in the technology used, particularly if it requires change in the operational procedure to move towards the achievement of the optimal split.

In order to better the understanding and interpretation of the results, a simulation model of the current "AS-IS" process will be modelled and compared to the generated "TO-BE" process according to the optimal split results obtained from the generated mathematical model. This has been useful in the visualisation of the effects of the optimal split on the throughput rate and utilization of the equipment in the studied distribution centre.

A number of alternative methods could have been used to aid in the validation of the results ranging from alternative Operations Research models to complex algorithms, however a simulation model fills a number of the loopholes that are commonly found when purely working from a theoretical standpoint; in particular the error of not being able to adequately identify faults in the modelling logic especially if the modeller has limited experience in the field. A simulation 
visually depicts a real-life process and is able to model potential processes which in turn enables the modeler to make comparisons between current and potential scenarios. This allows the modeller to easily identify whether or not the process flow is accurate as well as whether any bottlenecks or areas of concern develop over the running time of the evaluation period of the model.

The studied distribution centre along with the processes performed therein has been modelled to represent the real and current system used by the company. This along with the actual data that was obtained in order to model the processes aid in the overall validation of the results obtained in the simulation.

Furthermore, the model must be approved by the company to assure that the process flow is a close depiction of the true flow.

\section{Managerial implications}

Management will have the final decision as to whether the solution is implemented and the degree to which the solution is used within the distribution centre analysed. Management would need to put careful consideration into further feasibility studies and potential test runs if it is decided upon to implement the optimal split. It would also be advised that the company plans the implementation process to determine the timeline, client contract alterations and overall operational changes within the studied distribution centre to ensure a sound transition from the current to the optimal split.

Lastly, the company has future research opportunities following the conclusion of this study through the possible implementation of layout alternatives to improve the flow under the optimal split, and in the long-run to implement the modelling logic into each of the distribution centres within their network to ensure strategic alignment of flow and successful achievement of service level agreements.

\section{Recommendations}

The models constructed in order to meet the objective, scope and deliverables of this study have led to the ability to recommend that the company not only considers the implantation of the optimal parcel capacity split, but also conducts further feasibility studies to build a thorough business case in order to test run and potentially implement the results.

The recommendations resulting from the execution of this study are listed below:

- The distribution centre must convert current and future client contracts to conform to the optimal parcel capacity split.

- The number of forklifts in the studied distribution centre can be reduced aiding in a decrease in maintenance costs and increase in safety within the workplace.
- The company can reduce their current workforce within the distribution centre either through the process of procedurally and substantively fair retrenchment procedures or preferably through retraining or transferring to other distribution centres under the company's ownership.

\section{Future research opportunities}

The company should consider performing studies and evaluating the feasibility of the following study areas:

- The company could consider possible layout alternatives to improve the flow of the small, manual and forklift incompatible parcels which become less frequented parcels in the system following the change to the optimal split. There is a vast potential to design a layout that facilitates a more efficient flow for these parcel types.

- The company can also consider retraining workers who are no longer essential within the system in data analysis and worker management positions, so as to avoid higher retrenchment rates and to improve efficiency and effectiveness of the processes on the floor.

- The implementation of the logic obtained in this study could also be adapted to model each of the distribution centres within the company's network in order to strategically align the process flow and achievement of service level agreements.

\section{Conclusion}

This paper provides an outline of a problem that is faced by 3PL providers who process different parcel categories within their distribution centres. The fields of Operations Research and Simulation Modelling have been used as techniques to aid in the formulation of an optimal parcel category capacity split in order to ensure that the company maintains transparency and the ability to continuously deliver on customer expectations and prescribed service level agreements. The solution phase has been completed using two sub-models of the primary formulated mathematical model to obtain the parcel size category percentage split and the assignment of trucks to meet the demand on each of the analysed routes. A simulation model of the distribution centre then modelled the operations within the centre to validate whether or not the obtained optimal parcel size category percentage split is in fact a feasible solution and whether or not the solution obtained provided a better alternative to the current parcel size category percentage split. The analysis of the results provided insight into the potential implementation of the preferable chosen optimal split solution.

\section{Acknowledgements}

The authors are thankful to the referees for their valuable comments.

V.S.S. Yadavalli is thankful to NRF for their financial support. 


\section{References}

Armstrong \& Associates, 2013. Trends in 3PL/customer relationships - 2013. Wisconsin: Armstrong and Associates.

Briggs, E., Landry, T. \& Daugherty, P. 2010. 'Investigating the influence of velocity performance on satisfaction with third-party logistics service', Industrial Marketing Management, 39(4): 640649.

Business Dictionary. 2015. Third Party Logistics (3PL). [online] http://www.businessdictionary.com/definition/ third-partylogistics-3PL.html

Chu, Z. \& Wang, Q. 2012. 'Drivers of relationship quality in logistics outsourcing in China', Journal of Supply Chain Management, 48(3): 78-96.

El-Halwagi, M. 2006. Overview of optimization. In G. Stephanopoulos \& E. Pistikopoulos, eds. Process integration. 1st ed. London: Academic Press, 290-300.

Eyefortransport, 2013. Global chief supply chain officer strategy report, Amsterdam: Eyefortransport.

Eyefortransport, 2015. The 2015 3PL Rreport, Chicago: Eyefortransport.

Lieb, R. \& Bentz, B. 2005. 'The use of third-party logistics services by large American nanufacturers: The 2004 survey', Transportation Journal, 44(2): 5-12.

Maria, A. 1997. Introduction to mdelling and simulation. New York, State University of New York.

Masel, D. \& Goldsmith, D. 1997. Using a simulation model to evaluate the configuration of a sortation facility. Atlanta, s.n.

McWilliams, D., Stanfield, P. \& Geiger, C. 2005. 'The parcel hub scheduling problem: A simulation-based approach', Computer and Industrial Engineering, 49: 393-412.

McWilliams, D., Stanfield, P. \& Geiger, C. 2008. 'Minimising the completion time of the transfer operations in a central parcel consolidation terminal with unequal-batch-size inbound trailers', Computers and Industrial Engineering, 54(4): 709-720.

Robinson, S. 2014. The practice of model development and use. Palgrave: Macmillan.

Sargent, R.G. 2011. Verification and validation of simulation models. [online] http://www.informs-sim.org/wsc11 papers/016.pdf.

Tiwari, N. \& Shandilya, S. 2006. Operations research.. New Delhi: Printice Hall. 